\title{
INHALTSVERZEICHNIS
}

Geleitwort 9

Vorwort und Dank 11

1. LITERATURŮBERSICHT: 17

EIN KÜNSTLER ZWISCHEN DEN FRONTEN

$\begin{array}{ll}1.1 \text { Zeitgenössische Kunstkritik } & 17\end{array}$

1.2 Postume Veröffentlichungen in Russland und in der Sowjetunion 18

1.3 Postume Veröffentlichungen in Europa und in den Vereinigten Staaten 23

2. ZUM KÜNSTLERDASEIN BESTIMMT 31

2.1 Die ,Wanderjahre' 32

1872-75: München und Paris 33

1875-79: Abramtsevo, St. Petersburg, Kiew und Moskau 36

2.2 V. Serov. Selbst (1879) 40

2.3 1880: Studienbeginn 43

2.4 ,Infantin' und ,Amazone': Bildnisse Liudmila und Maria Mamontova (1884) 46

2.5 Der forschende Blick. Serovs frühe Selbstbildnisse 51

3. AUF DER SUCHE NACH EIGENER BILDSPRACHE: 55 SEROVS STILISTISCHE EVOLUTION NACH

SEINEN REISEN ZWISCHEN 1885 UND 1887. EINE FESTSTELLUNG

3.1 Europa-Reise von 1885: Deutschland, Holland, Belgien 55

3.2 Zurück in Russland: Experimentieren, Wagen, Scheitern 57

3.3 Das erste Meisterwerk: Das Bildnis Olga Trubnikova (1886) 59 als metaphysische Lichtinszenierung

3.4 Ein Professor ohne Diplom $\quad 70$

3.5 1887: Italien und die Folgen $\quad 72$

Vera Mamontova (1887) 73

Maria Simonovich (1888) 76

Von Künstlern bewundert. Von Kritikern zerrissen 78

Frage des Impressionismus 83 
4. WEGE ZUM BILD: UNTERSUCHUNG UND STILISTISCHEN EVOLUTION

4.1 Serovs Ideenaustausch mit llya Repin, Mikhail Vrubel und Konstantin Korovin 89

Ilya Repin (1844-1930)

Mikhail Vrubel (1856-1910)

Konstantin Korovin (1861-1939)

4.2 Von Angesicht zu Angesicht: Serovs Begegnungen mit den Werken 98 zeitgenössischer europäischer Maler in den russischen Sammlungen

Die Kushelevskaia Galerie

Sammlung Dmitry Botkin

Sammlung Sergei Tretyakov

Sammlung llya Ostroukhov

Sammlung Nikolai Kuznetsov

4.3 Dem Künstler auf der Spur. Zu den Stationen 110

von Serovs Europa-Reisen von 1885 und 1887

Karl Koepping und seine Rolle in Serovs Europa-Reise von 1885

Ein Tag - eine Weltreise: Serov in der Antwerpener Weltausstellung von 1885

Die neuen Horizonte: Sehnsuchtsort Italien $\quad 118$

Venedig als Inspirationsstätte der internationalen Moderne $\quad 120$

Florenz und Mailand zwischen Vergangenheit und Gegenwart 131

Die Bildnisse von Vera Mamontova (1887) und Maria Simonovich (1888) 135

im Zeichen transalpiner Kunst

4.4 Serovs moderne Unmittelbarkeit

... im Nachklang impressionistischer Stimmung

... am Beispiel seiner Kinderbildnisse

... in den Auftragsporträts des Herrscherhauses und Hochadels

\section{AUSSTELLUNGSBETEILIGUNGEN AB 1889}

5.1 Die ,erste' Parisreise: Exposition Universelle von 1889

Französische Kunst

Die Vereinigten Staaten

Deutschlands unabhängige Abteilung von Karl Koepping, Max Liebermann und Gotthard Kuehl Niederlande, Italien, Dänemark und Norwegen

Russland

5.2 Serov in Deutschland und Österreich: Reiseaufenthalte und Ausstellungen 202 Sergei Diaghilevs Ausstellung in der Münchner Secession und im Salon Schulte (1898) 208 und die Reaktionen deutscher Kritiker

5.3 Serov in Skandinavien

5.4 Serov in Frankreich (1900, 1906), Deutschland (1906-07) und Italien (1907) 215

Die Exposition Universelle von 1900 und Serovs Stellung innerhalb

der russischen Abteilung im Licht der internationalen Kunstkritik Serov im Salon d'Automne (1906), im Salon Schulte (1906-07) und auf der Biennale di Venezia (1907)

5.5 Anmerkungen zu Serovs Mitwirkung in Sergei Diaghilevs

Mir iskusstva und Ballets Russes 
6. LINIE, FARBE UND FORM:

TRADITION UND NEUERUNG UM 1900

$\begin{array}{ll}\text { 6.1 Die Farbe Schwarz } & 253\end{array}$

Savva Mamontov (1887/90), die Abramtsevo-Künstlerkolonie und 253

die erste Russische Privatoper
Angelo Masini (1890), Francesco Tamagno (1891) und die Alten Meister

$\begin{array}{ll}\text { Isaak Levitan (1893) } & 265\end{array}$

$\begin{array}{ll}\text { Mara Oliv (1895) } & 268\end{array}$

$\begin{array}{ll}\text { Mikhail Morozov (1902) } & 270\end{array}$

$\begin{array}{ll}\text { 6.2 Valentin Serov und Anders Zorn } & 278\end{array}$

Nikolai Rimsky-Korsakov (1898) 280

Nikolai II. (1900), Großherzog Mikhail Nikolaievich (1900), Pavel Kharitonenko (1901), 282

Alexei Goriainov (1901), llya Ostroukhov (1902) und Evdokia Loseva (1903)

6.3 Die Frage des non-finito in Serovs Fuvre: Der Fall Konstantin Korovin (1891) 284

6.4 Von tonalen Farbsymphonien zu Farbkontrasten und Klarheit der Form 290

Sophia Botkina (1899) $\quad 291$

Herzogin Zinaida Yusupova (1900-02) 296

Henrietta Hirschman (1906, 1907 und 1911) 302

Maria Akimova (1908), Nikolai Pozniakov (1908), Ivan Morozov (1910) 312

Herzogin Olga Orlova (1911) 314

6.5 Silhouette, Linie, Fläche: Die Suche nach einer neuen Form- und Farbsprache 319

Serovs Beschäftigung mit der Antike und seine Reise nach Griechenland (1907): Odysseus 320

und Nausikaa (1909-10), Raub der Europa (1909-10) und Metamorphosen von Ovid (1910-11)

Ida Rubinstein (1910) im Kontext japanischer Kunst

7. NACHWORT: VALENTIN SEROV EIN ALTMEISTERLICH MODERNER KÜNSTLER

FARBTAFELN

Anhang

Valentin Serovs Liste: Seine besten Werke $\quad 375$

$\begin{array}{ll}\text { Valentin Serovs Künstlervita } & 376\end{array}$

$\begin{array}{ll}\text { Bibliografie } & 403\end{array}$

$\begin{array}{lr}\text { Namensregister } & 419\end{array}$

$\begin{array}{ll}\text { Bildnachweis } & 435\end{array}$ 
